\title{
Optimal Adjustment Algorithm for $p$ Coordinates and the Starting Point in Interior Point Methods
}

\author{
Carla T. L. S. Ghidini ${ }^{1}$, Aurelio R. L. Oliveira ${ }^{1}$, Jair Silva ${ }^{2}$ \\ ${ }^{1}$ Institute of Mathematics, Statistics and Scientific of Computation (IMECC), \\ State University of Campinas (UNICAMP), São Paulo, Brazil \\ ${ }^{2}$ Federal University of Mato Grosso do Sul (UFMS), Mato Grosso do Sul, Brazil \\ E-mail: \{carla,aurelio\}@ime.unicamp.br,jairmt@gmail.com \\ Received September 15, 2011; revised October 16, 2011; accepted October 30, 2011
}

\begin{abstract}
Optimal adjustment algorithm for $p$ coordinates is a generalization of the optimal pair adjustment algorithm for linear programming, which in turn is based on von Neumann's algorithm. Its main advantages are simplicity and quick progress in the early iterations. In this work, to accelerate the convergence of the interior point method, few iterations of this generalized algorithm are applied to the Mehrotra's heuristic, which determines the starting point for the interior point method in the PCx software. Computational experiments in a set of linear programming problems have shown that this approach reduces the total number of iterations and the running time for many of them, including large-scale ones.
\end{abstract}

Keywords: Von Neumann's Algorithm, Mehrotra's Heuristic, Interior Point Methods, Linear Programming

\section{Introduction}

In 1948, von Neumann proposed to Dantzig an algorithm for finding a feasible solution to a linear program with a convexity constraint recast in the form $([1,2])$ :

$$
P x=0, e^{t} x=1, \quad x \geq 0
$$

where $P \in \mathrm{R}^{m \times n}, x \in \mathrm{R}^{n}, e \in \mathrm{R}^{n}$ is the vector of all ones, and the columns of $P$ have norm one, i.e., $\left\|P_{j}\right\|=1$, for $j=1, \cdots, n$.

This algorithm, which was later studied by Epelman and Freund [3], has interesting properties such as simplicity and fast initial advance. However, it is not very practical for solving linear problems because its convergence rate is slow.

Gonçalves [4] presented four new algorithms based on von Neumann's algorithm and among them the optimal pair adjustment algorithm has the best performance in practice.

The optimal pair adjustment algorithm inherits the good properties of von Neumann's algorithm. Although it is proved in [5] that this algorithm is faster than von Neumann's algorithm, nevertheless, it is impractical to solve linear programming problems, because its convergence rate is also very slow.

In [6], the idea presented by Gonçalves, Storer and
Gondzio [5] to develop the optimal pair adjustment algorithm was generalized for $p$ coordinates and then the optimal adjustment algorithm for $p$ coordinates arose. The value of $p$ is bounded by the number of variables of the problem.

The new algorithm is not suitable to solve linear programming problems until achieving optimality. Thus, the idea is to exploit its simplicity and quick initial progress during the early iterations and to use it together with an interior point method to accelerate convergence.

Knowing that the starting point greatly influences the performance of the interior point method, in this work the optimal adjustment algorithm for $p$ coordinates is applied within the Mehrotra's heuristic [7], which determines the starting point for the method interior points in the PCx software, so that the starting points can be obtained even better. This approach is different to the classical warm-starting approach, which uses a known solution for some problem (e.g., relaxation in the columngeneration) to define a new starting point for a closely related problem or perturbed problem instance ([8-10]).

The paper is organized as follows. In Section 2, the definition of the problem is given and von Neumann's algorithm is described. In Section 3, the optimal pair adjustment algorithm is recalled. In Section 4, the optimal adjustment algorithm for $p$ coordinates is proposed 
and some theoretical results are described. Section 5 discusses warm-starting in interior point methods. Numerical experiments are shown in Section 6. In Section 7, the conclusions are drawn and future perspectives are suggested.

\section{Von Neumann's Algorithm}

Considering the problem of finding a feasible solution of the set of linear constraints (1.1), geometrically, the columns $P_{j}$ can be viewed as points lying on the $m$-dimensional hypersphere with unit radius and center at the origin. This problem can be described as assigning nonnegative weights $x_{j}$ to columns $P_{j}$ in such a way that after being re-scaled its center of gravity is the origin.

First, the algorithm finds the column $P_{s}$ of $P$ which makes the largest angle with the residue $b^{k-1}=P x^{k-1}$ and then the next residual $b^{k}$ is given by projecting the origin on the line segment connecting $b^{k-1}$ to $P_{s}$. See Figure 1.

\section{VON NEUMANN'S ALGORITHM}

Given: $x^{0} \geq 0$, with $e^{t} x^{0}=1$. Compute $b^{0}=P x^{0}$. $k=1$

Do

\{

1. Compute:

$$
\begin{aligned}
& s=\arg \min _{j=1, \cdots, n}\left\{P_{j}^{t} b^{k-1}\right\} . \\
& v^{k-1}=P_{s}^{t} b^{k-1}
\end{aligned}
$$

2. If $v^{k-1}>0$, then STOP. The problem (1.1) is infeasible.

3. Compute:

$$
\begin{aligned}
& u^{k-1}=\left\|b^{k-1}\right\|, \\
& \lambda=\frac{1-v^{k-1}}{\left(u^{k-1}\right)^{2}-2 v^{k-1}+1} .
\end{aligned}
$$

4. Update:

$$
\begin{aligned}
& b^{k}=\lambda b^{k-1}+(1-\lambda) P_{s}, \\
& x^{k}=\lambda x^{k-1}+(1-\lambda) e_{s} .
\end{aligned}
$$

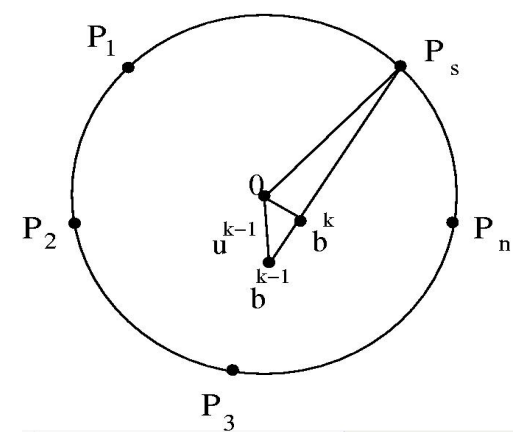

Figure 1. Illustration of von Neumann's algorithm. where $e_{s}$ is the vector of the canonical basis with 1 in position $s$.

5. Stopping Criterion:

$$
\begin{aligned}
& \quad E^{k}=\left\|b^{k}-b^{k-1}\right\| /\left\|b^{k}\right\| \\
& k=k+1 . \\
& \text { \} while } \left.\left(E^{k}>\varepsilon\right)\right\},
\end{aligned}
$$

where $\varepsilon$ is a predetermined percentage.

Note that $b^{k}=P x^{k}$, for all $k \geq 0$. The initial approximation $x^{0}$ is arbitrary, since $e^{t} x^{0}=1$, then $x_{j}=1 / n$ for $j=1, \cdots, n$ was used. For any given iteration $k$ column $P_{s}$ is the column which makes the largest angle with the vector $b^{k-1}$. Furthermore, if $v^{k-1}=P_{s}^{t} b^{k-1} \neq 0$, then $0<1$ $-v^{k-1}<\left(u^{k-1}\right)^{2}-2 v^{k-1}+1$ thus, $0<\lambda<1$. Moreover, the new residual is smaller than the previous one, i.e., $u^{k}<$ $u^{k-1}$, as can easily be seen in Figure 1, the triangle $0 b^{k-1} b^{k}$ has hypotenuse $u^{k-1}=0 b^{k-1}$ and side $u^{k}=0 b^{k}$.

The effort per iteration of von Neumann's algorithm is dominated by matrix-vector multiplication needed when selecting column $P_{s}$ which is $O(n z(P))$ where $n z(P)$ is the number of the entries of $P$. This effort can be reduced significantly as the matrix $P$ is sparse. For more details of this algorithm see [11,12].

\section{Optimal Pair Adjustment Algorithm}

In his $\mathrm{PhD}$ thesis [4], Gonçalves studied von Neumann's algorithm and introduced four new algorithms based on it. Among them, emphasis is given to the weight-reduction algorithm and the optimal pair adjustment algorithm. The optimal pair adjustment algorithm was the one that performed better in practice.

The weight-reduction algorithm was proposed as an attempt to improve the efficiency of von Neumann's algorithm. It is based on the idea that the residual $b^{k-1}$ can be moved closer to origin 0 , increasing the weight $x_{j}$ for a given column $P_{j}$ and decreasing the weight $x_{i}$ for another column $P_{i}$.

In particular, it is expected that the new residual $b^{k}$ is closer to origin 0 than the residual $b^{k-1}$ if the weight in column $P_{s^{+}}$is increased when $P_{s^{+}}$has the largest angle with the residual $b^{k-1}$ and the weight in column $P_{s^{-}}$is decreased when $P_{s^{+}}$has the smallest angle with the residual $b^{k-1}$.

This corresponds to making residual $b^{k-1}$ move in the direction $P_{s^{+}}-P_{s^{-}}$. The new residual $b^{k}$ is the point that minimizes the distance from origin 0 to this line.

Notice that the minimization of this distance is subject to the maximum possible decrease of $x_{s^{-}}$. Since $x_{j} \geq 0$ for all $j$, then $x_{s^{-}}$can be decreased until it vanishes. Figure 2 is a geometric illustration of how the algorithm works by iteration. 


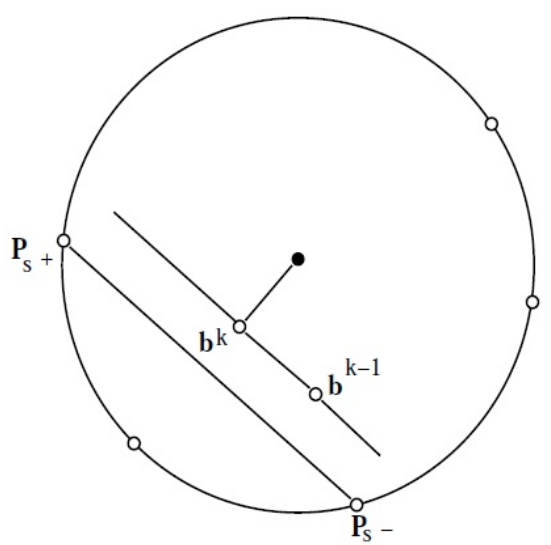

Figure 2. Illustration of weight-reduction algorithm.

\subsection{Optimal Pair Adjustment Algorithm}

The optimal pair adjustment algorithm is a generalization of the weight-reduction algorithm designed to give the maximum possible freedom to two of the weights $x_{j}$ (see [5]). In a way, we can say that it prioritizes only two variables by iteration because it finds the optimal value for two coordinates and adjusts the remaining coordinates according to these values.

Similar to the weight-reduction algorithm, the optimal pair adjustment algorithm starts by identifying vectors $P_{s^{+}}$and $P_{s^{-}}$, which make the largest and smallest angles with the vector $b^{k-1}$. Afterwards, values $x_{s+}^{k}, x_{s-}^{k}, \lambda$ are found, where $x_{j}^{k}=\lambda x_{j}^{k-1}$ for all $j \neq s+$ and $j \neq s-$. These values minimize the distance between $b^{k}$ and the origin while satisfying the convexity and the non-negativity constraints. The solution of this optimization problem is easily computed examining the KarushKuhn-Tucher conditions (KKT).

The main difference between the weight-reduction algorithm and the optimal pair adjustment algorithm is that only the weights of $P_{s^{+}}$and $P_{s^{-}}$are changed in the first algorithm while in the second algorithm all other weights are also changed.

\section{OPTIMAL PAIR ADJUSTMENT ALGORITHM}

Given: $x^{0} \geq 0$, with $e^{t} x^{0}=1$. Compute $b^{0}=P x^{0}$. $k=1$

Do

\{

1. Compute:

$$
\begin{aligned}
& s^{+}=\arg \min _{j=1, \ldots, n}\left\{P_{j}^{t} b^{k-1}\right\}, \\
& s^{-}=\arg \max _{j=1, \ldots, n}\left\{P_{j}^{t} b^{k-1} / x_{j}>0\right\}, \\
& v^{k-1}=P_{s+}^{t} b^{k-1}
\end{aligned}
$$

2. If $v^{k-1}>0$, then STOP. The problem (1.1) is infeasible.
3. Solve the problem:

$$
\begin{array}{ll}
\min \|\bar{b}\|^{2} \\
\text { s.t. } & \lambda_{0}\left(1-x_{s+}^{k-1}-x_{s-}^{k-1}\right)+\lambda_{1}+\lambda_{2}=1, \\
& \lambda_{i} \geq 0, \text { for } i=0,1,2 .
\end{array}
$$

where

$$
\bar{b}=\lambda_{0}\left(b^{k-1}-x_{s+}^{k-1} P_{s+}-x_{s-}^{k-1} P_{s-}\right)+\lambda_{1} P_{s+}+\lambda_{2} P_{s-} .
$$

4. Update:

$$
\begin{aligned}
b^{k} & =\lambda_{0}\left(b^{k-1}-x_{s+}^{k-1} P_{s+}-x_{s-}^{k-1} P_{s-}\right)+\lambda_{1} P_{s+}+\lambda_{2} P_{s-}, \\
u^{k} & =\left\|b^{k}\right\|, \\
x^{k} & = \begin{cases}\lambda_{0} x_{j}^{k-1}, & j \neq s^{+} \\
\lambda_{1}, & j=s^{+} \\
\lambda_{2}, & j=s^{-}\end{cases}
\end{aligned}
$$

\section{Stopping Criterion:}

$$
\begin{aligned}
& E^{k}=\left\|b^{k}-b^{k-1}\right\| /\left\|b^{k}\right\| \\
& k=k+1 .
\end{aligned}
$$

\} while $\left.\left(E^{k}>\varepsilon\right)\right\}$.

In iteration $k$, column $P_{s^{+}}$is the column which makes the largest angle with the vector $b^{k-1}$ and column $P_{s^{-}}$is the column which makes the smallest angle with the vector $b^{k-1}$ such that $x_{s}>0$.

\subsection{Subproblem Solution}

In order to solve subproblem (3.2), first the subproblem is rewritten as:

$$
\begin{array}{ll}
\min & \|\bar{b}\|^{2} \\
\text { s.t. } & 1-\lambda_{1}-\lambda_{2} \geq 0, \\
& \lambda_{i} \geq 0, \text { for } i=0,1,2 .
\end{array}
$$

where,

$$
\begin{aligned}
\bar{b}= & \frac{1-\lambda_{1}-\lambda_{2}}{\left(1-x_{s+}^{k-1}-x_{s-}^{k-1}\right)}\left(b^{k-1}-x_{s+}^{k-1} P_{s+}-x_{s-}^{k-1} P_{s-}\right)+\lambda_{1} P_{s+} \\
& +\lambda_{2} P_{s-} .
\end{aligned}
$$

Define:

$$
g_{0}\left(\lambda_{1}, \lambda_{2}\right)=\lambda_{1}+\lambda_{2}-1, g_{1}\left(\lambda_{1}, \lambda_{2}\right)=-\lambda_{1}, g_{2}\left(\lambda_{1}, \lambda_{2}\right)=-\lambda_{2} \text {. }
$$

Denote the objective function by $f\left(\lambda_{1}, \lambda_{2}\right)$ and let $\left(\bar{\lambda}_{1}, \bar{\lambda}_{2}\right)$ be a feasible solution.

Considering the convexity of the objective function and constraints, if $\left(\bar{\lambda}_{1}, \bar{\lambda}_{2}\right)$ is an optimal local solution then it is also an optimal global solution. Thus, $\left(\bar{\lambda}_{1}, \bar{\lambda}_{2}\right)$ met the KKT constraints given by:

$$
\nabla f\left(\lambda_{1}, \lambda_{2}\right)+\sum_{i=0}^{2} \mu_{i} \nabla g_{i}\left(\lambda_{1}, \lambda_{2}\right)=0 .
$$




$$
\begin{array}{ll}
g_{i}\left(\lambda_{1}, \lambda_{2}\right) \leq 0, & \text { for } i=0,1,2 . \\
\mu_{i} g_{i}\left(\lambda_{1}, \lambda_{2}\right)=0, & \text { for } i=0,1,2 . \\
\mu_{i} \geq 0, & \text { for } i=0,1,2 .
\end{array}
$$

where $\mu_{i}$ are Lagrange multipliers.

Problem (3.3) is solved by selecting a feasible solution among all possibilities that meet the KKT conditions. This is done by analyzing the following cases:
a) $\lambda_{1}=\lambda_{2}=0$;
b) $\lambda_{1}=0 ; 0<\lambda_{2}<1$;
c) $\lambda_{1}=0 ; \lambda_{2}=1$;
d) $0<\lambda_{1}<1 ; \lambda_{2}=0$;
e) $0<\lambda_{1}<1 ; 0<\lambda_{2}<1 ; \lambda_{1}+\lambda_{2}-1=0$;
f) $\lambda_{1}=1 ; \lambda_{2}=0$;
g) $0<\lambda_{1}<1 ; 0<\lambda_{2}<1 ; \lambda_{1}+\lambda_{2}-1=0$;

For each case above, the known values are replaced in the KKT equations and the resulting linear system is solved.

\section{Optimal Adjustment Algorithm for $p$ Coordinates}

The optimal adjustment algorithm for $p$ coordinates is developed generalizing the ideas used in [5] for the optimal pair adjustment algorithm. Instead of only two columns to be used to formulate the problem, any amount of columns can be used and thus, importance will be given to any desired amount of variables.

The strategy to prioritize the variables is free. In this work, we chose to take $p / 2$ columns making the largest angle with the vector $b^{k}$ and the remaining $p / 2$ columns that make the smallest angle with the vector $b^{k}$. If $p$ is odd then one more column is added to the set of vectors that make the largest angle with the vector $b^{k}$, for example.

The structure of the optimal adjustment algorithm for $p$ coordinates is similar to the optimal pair adjustment algorithm. It begins by identifying the $s_{1}$ columns that make the largest angle with the vector $b^{k-1}$, then $s_{2}$ columns that make the smallest angle with the vector $b^{k-1}$ are determined, where $s_{1}+s_{2}=p$ and $p$ is the number of columns that are prioritized. Next, the optimization subproblem is solved and the residual and the current point are updated.

\section{OPTIMAL ADJUSTMENT ALGORITHM FOR $p$} COORDINATES

Given: $x^{0} \geq 0$, with $e^{t} x^{0}=1$. Compute $b^{0}=P x^{0}$. $k=1$

Do

\{

\section{Compute:}

$\left\{\mathrm{P}_{\eta_{1}^{+}}, \cdots, \mathrm{P}_{\eta_{s_{1}}^{+}}\right\}$, which make the largest angle with the vetor $b^{k-1}$.

$\left\{\mathrm{P}_{\eta_{1}^{-}}, \cdots, \mathrm{P}_{\eta_{s_{1}}^{-}}\right\}$, which make the smallest angle with the vector $b^{k-1}$ and such that $x_{i}^{k-1}>0$, where $s_{1}+s_{2}$ $=p$.

$$
v^{k-1}=\text { minimum }{ }_{i=1, \cdots, s_{1}}\left\{P_{\eta_{i}^{t}}^{t} b^{k-1}\right\}
$$

2. If $v^{k-1}>0$, then STOP. The problem (1.1) is infeasible.

3. Solve the problem:

$$
\begin{array}{ll}
\min \|\bar{b}\|^{2} & \\
\text { s.t. } & \lambda_{0}\left(1-\sum_{i=1}^{s_{1}} x_{\eta_{i}^{+}}^{k-1}-\sum_{j=1}^{s_{2}} x_{\eta_{j}^{-}}^{k-1}\right)+\sum_{i=1}^{s_{1}} \lambda_{\eta_{i}^{+}}+\sum_{j=1}^{s_{2}} \lambda_{\eta_{j}^{-}}=1, \\
& \lambda_{0} \geq 0, \\
& \lambda_{\eta_{i}^{+}} \geq 0, \text { for } i=1, \cdots, s_{1}, \\
& \lambda_{\eta_{j}^{-}} \geq 0, \text { for } j=1, \cdots, s_{2} .
\end{array}
$$

where

$$
\begin{aligned}
\bar{b}= & \lambda_{0}\left(b^{k-1}-\sum_{i=1}^{s_{1}} x_{\eta_{i}^{+}}^{k-1} P_{\eta_{i}^{+}}-\sum_{j=1}^{s_{2}} x_{\eta_{j}^{-}}^{k-1} P_{\eta_{j}^{-}}\right)+\sum_{i=1}^{s_{1}} \lambda_{\eta_{i}^{+}} P_{\eta_{i}^{+}} \\
& +\sum_{j=1}^{s_{2}} \lambda_{\eta_{j}^{-}} P_{\eta_{j}^{-}}
\end{aligned}
$$

4. Update:

$$
\begin{aligned}
b^{k}= & \lambda_{0}\left(b^{k-1}-\sum_{i=1}^{s_{1}} x_{\eta_{i}^{+}}^{k-1} P_{\eta_{i}^{+}}-\sum_{j=1}^{s_{2}} x_{\eta_{j}^{-}}^{k-1} P_{\eta_{j}^{-}}\right)+\sum_{i=1}^{s_{1}} \lambda_{\eta_{i}^{+}} P_{\eta_{i}^{+}} \\
& +\sum_{j=1}^{s_{2}} \lambda_{\eta_{j}^{-}} P_{\eta_{j}^{-}}, \\
u^{k}= & \left\|b^{k}\right\|, \\
x_{j}^{k}= & \begin{cases}\lambda_{0} x_{j}^{k-1}, & j \notin\left\{\eta_{1}^{+}, \cdots, \eta_{s_{1}}^{+}, \eta_{1}^{-}, \cdots, \eta_{s_{2}}^{-}\right\}, \\
\lambda_{\eta_{i}^{+}}, & j=\eta_{i}^{+} ; i=1, \cdots, s_{1}, \\
\lambda_{\eta_{j}^{-}}, & j=\eta_{j}^{-} ; j=1, \cdots, s_{2} .\end{cases}
\end{aligned}
$$

5. Stopping Criterion:

$$
\begin{aligned}
& \quad E^{k}=\left\|b^{k}-b^{k-1}\right\| /\left\|b^{k}\right\| \\
& k=k+1 . \\
& \text { \} while } \left.\left(E^{k}>\varepsilon\right)\right\} .
\end{aligned}
$$

In Step 2 of the algorithm, if $v^{k-1}>0$ then all columns $P_{j}$ of matrix $P$ are on the same side of the hyper-plane through the origin and perpendicular to direction $b^{k-1}$. This means that any convex combination of the columns $P_{j}$ resulting in the origin can be found. In this case, the 
problem is infeasible.

\subsection{Subproblem Solution Using Interior Point Methods}

For each iteration of the optimal adjustment algorithm for $p$ coordinates, it is necessary to solve subproblem (4.4). This subproblem is solved finding a solution in the positive orthant, subject to a linear equation system of order $(p+1)$. A way to solve it is to verify all possible feasible solutions as in the pair adjustment algorithm. The drawback that comes naturally in solving the subproblem in this way is that the number of possible cases to be verified grows exponentially with the value of $p$ as shown next.

In fact, to solve the subproblem (4.4) first the variable $\lambda_{0}$, as defined in (4.5), is removed from the problem.

$$
\lambda_{0}=\frac{1-\sum_{i=1}^{s_{1}} \lambda_{\eta_{i}^{+}}-\sum_{j=1}^{s_{2}} \lambda_{\eta_{j}^{-}}}{1-\sum_{i=1}^{s_{1}} x_{\eta_{i}^{+}}^{k-1}-\sum_{j=1}^{s_{2}} x_{\eta_{j}^{k}}^{k-1}}
$$

Thus, the subproblem (4.4) was rewritten as:

$$
\begin{array}{ll}
\min \|\bar{b}\|^{2} & \\
\text { s.t. } & 1-\sum_{i=1}^{s_{1}} \lambda_{\eta_{i}^{+}}-\sum_{j=1}^{s_{2}} \lambda_{\eta_{j}^{-}} \geq 0, \\
& \lambda_{\eta_{i}^{+}} \geq 0, \text { for } i=1, \cdots, s_{1}, \\
& \lambda_{\eta_{j}^{-}} \geq 0, \text { for } j=1, \cdots, s_{2} .
\end{array}
$$

where

$$
\begin{aligned}
\bar{b}= & \lambda_{0}\left(b^{k-1}-\sum_{i=1}^{s_{1}} x_{\eta_{i}^{+}}^{k-1} P_{\eta_{i}^{+}}-\sum_{j=1}^{s_{2}} x_{\eta_{j}^{-}}^{k-1} P_{\eta_{j}^{-}}\right)+\sum_{i=1}^{s_{1}} \lambda_{\eta_{i}^{+}} P_{\eta_{i}^{+}} \\
& +\sum_{j=1}^{s_{2}} \lambda_{\eta_{\bar{j}}^{-}} P_{\eta_{\bar{j}}^{-}}
\end{aligned}
$$

Defining:

$$
\begin{gathered}
g_{0}\left(\lambda_{\eta_{1}^{+}}, \cdots, \lambda_{\eta_{s_{1}^{+}}}, \lambda_{\eta_{1}^{-}}, \cdots, \lambda_{\eta_{s_{2}}^{-}}\right)=\sum_{i=1}^{s_{1}} \lambda_{\eta_{i}^{+}}+\sum_{j=1}^{s_{2}} \lambda_{\eta_{j}^{-}}-1 \\
g_{i}\left(\lambda_{\eta_{1}^{+}}, \cdots, \lambda_{\eta_{s_{1}^{+}}}, \lambda_{\eta_{1}^{-}}, \cdots, \lambda_{\eta_{s_{2}}^{-}}\right)=-\lambda_{\eta_{i}^{+}}, i=1, \cdots, s_{1} \\
h_{j}\left(\lambda_{\eta_{1}^{+}}, \cdots, \lambda_{\eta_{s_{1}^{+}}}, \lambda_{\eta_{1}^{-}}, \cdots, \lambda_{\eta_{s_{2}}^{-}}\right)=-\lambda_{\eta_{j}^{-}}, j=1, \cdots, s_{2}
\end{gathered}
$$

and denoting the objective function by $f\left(\lambda_{\eta_{1}^{+}}, \cdots, \lambda_{\eta_{s_{1}}^{+}}, \lambda_{\eta_{1}^{-}}, \cdots, \lambda_{\eta_{s_{2}}^{-}}\right)$, the KKT conditions of the subproblem are given by:

$$
\left\{\begin{array}{l}
\nabla f\left(\lambda_{\eta_{1}^{+}}, \cdots, \lambda_{\eta_{s_{1}}^{+}}, \lambda_{\eta_{1}^{-}}, \cdots, \lambda_{\eta_{s_{2}}^{-}}\right) \\
+\sum_{i=0}^{s_{1}} \mu_{g_{i}} \nabla g_{i}\left(\lambda_{\eta_{1}^{+}}, \cdots, \lambda_{\eta_{s_{1}}^{+}}, \lambda_{\eta_{1}^{-}}, \cdots, \lambda_{\eta_{s_{2}}^{-}}\right) \\
+\sum_{j=0}^{s_{2}} \mu_{h_{j}} \nabla h_{j}\left(\lambda_{\eta_{1}^{+}}, \cdots, \lambda_{\eta_{s_{1}}^{+}}, \lambda_{\eta_{1}^{-}}, \cdots, \lambda_{\eta_{s_{2}}^{-}}\right)=0, \\
\mu_{g_{i}} g_{i}\left(\lambda_{\eta_{1}^{+}}, \cdots, \lambda_{\eta_{s_{1}}^{+}}, \lambda_{\eta_{1}^{-}}, \cdots, \lambda_{\eta_{s_{2}}^{-}}\right)=0, \text { for } i=0, \cdots, s_{1}, \\
\mu_{h_{j}} h_{j}\left(\lambda_{\eta_{1}^{+}}, \cdots, \lambda_{\eta_{s_{1}}^{+}}, \lambda_{\eta_{1}^{-}}, \cdots, \lambda_{\eta_{s_{2}}^{-}}\right)=0, \text { for } j=1, \cdots, s_{2}, \\
\mu_{g_{i}} \geq 0, \text { for } i=0, \cdots, s_{1}, \\
\mu_{h_{j}} \geq 0, \text { for } j=1, \cdots, s_{2}, \\
g_{i}\left(\lambda_{\eta_{1}^{+}}, \cdots, \lambda_{\eta_{s_{1}}^{+}}, \lambda_{\eta_{1}^{-}}, \cdots, \lambda_{\eta_{s_{2}}^{-}}\right) \leq 0, \text { for } i=0, \cdots, s_{1}, \\
h_{j}\left(\lambda_{\eta_{1}^{+}}, \cdots, \lambda_{\eta_{s_{1}}^{+}}, \lambda_{\eta_{1}^{-}}, \cdots, \lambda_{\eta_{s_{2}}^{-}}\right) \leq 0, \text { for } j=1, \cdots, s_{2} .
\end{array}\right.
$$

Then, the subproblem (4.6) is solved by selecting a feasible solution among all the possibilities that satisfy the KKT conditions (4.7). For this, all possible cases of possible values for the variables $\lambda_{\eta_{i}^{+}}, i=1, \cdots, s_{1}$, and $\lambda_{\eta_{\bar{j}}^{-}}, j=1, \cdots, s_{2}$, must be analyzed. The cases to be considered are:

- $\quad$ Case: $\lambda_{\eta_{i}^{+}}=0, i=1, \cdots, s_{1}$ and $\lambda_{\eta_{j}^{-}}=0$, $j=1, \cdots, s_{2}$,

- $\quad$ Case: one $\lambda_{k} \neq 0$, with $k \notin\left\{\eta_{1}^{+}, \cdots, \eta_{s_{1}}^{+}, \eta_{1}^{-}, \cdots, \eta_{s_{2}}^{-}\right\}$ and others equal to zero, which gives a combination of $C_{1}^{p}$ cases, but again we have to consider the case where $\lambda_{k}=1$ and $\lambda_{k} \neq 1$. Thus, we have $2 C_{1}^{p}$ possibilities.

- Case: $\lambda_{i} \neq 0$ and $\lambda_{j} \neq 0$, and

$i, j \in\left\{\eta_{1}^{+}, \cdots, \eta_{s_{1}}^{+}, \eta_{1}^{-}, \cdots, \eta_{s_{2}}^{-}\right\}$and others equal to zero, which gives a combination of $C_{2}^{p}$ cases, but again we have to consider the case where

$\lambda_{i}+\lambda_{j}=1$ and $\lambda_{i}+\lambda_{j} \neq 1$. Thus, we have $2 C_{2}^{p}$ possibilities.

The total number of cases when $p$ coordinates are modified is:

$$
1+2 C_{1}^{p}+2 C_{2}^{p}+2 C_{3}^{p}+\cdots+2 C_{p}^{p}=2^{(p+1)}-1
$$

Therefore, this strategy is inefficient even for values of $p$ which are not too large.

In order to deal with such a difficulty, the subproblem (4.4) is approached differently and solved using interior point methods. This is done as follows:

First, the subproblem is rewritten in matrix form: 


$$
\begin{array}{cl}
\min & \frac{1}{2}\|W \lambda\|^{2} \\
\text { s.t. } & a^{t} \lambda=1, \\
& \lambda \geq 0,
\end{array}
$$

where

$$
\begin{aligned}
& W=\left[\bar{w} P_{\eta_{1}^{+}} \cdots P_{\eta_{s_{1}}^{+}} P_{\eta_{1}^{-}} \cdots P_{\eta_{s_{2}}^{-}}\right] \\
& \bar{w}=b^{k-1}-\sum_{i=1}^{s_{1}} x_{\eta_{i}^{+}}^{k-1} P_{\eta_{i}^{+}}-\sum_{j=1}^{s_{2}} x_{\eta_{j}^{-}}^{k-1} P_{\eta_{j}^{-}}, \\
& \lambda^{t}=\left(\lambda_{0}, \lambda_{\eta_{1}^{+}}, \cdots, \lambda_{\eta_{s_{1}^{+}}^{+}}, \lambda_{\eta_{1}^{-}}, \cdots, \lambda_{\eta_{s_{2}}^{-}}\right), \\
& a=\left(a_{1} 1 \cdots 1\right)^{t}, a_{1}=1-\sum_{i=1}^{s_{1}} x_{\eta_{i}^{+}}^{k-1}-\sum_{j=1}^{s_{2}} x_{\eta_{j}^{-}}^{k-1}
\end{aligned}
$$

The KKT equations of problem (4.8) are given by:

$$
W^{t} W \lambda+a \gamma-\mu=0, \mu^{t} \lambda=0, a^{t} \lambda-1=0, \lambda \geq 0, \mu \geq 0 \text {. }
$$

where, $\gamma$ and $\mu$ are Lagrange multipliers of equality and inequality constraints respectively and $(p+1) \times(p+1)$ is the dimension of the matrix $W^{t} W$.

Now the interior point method is applied to those equations.

The linear system arising at each iteration of the interior point method applied to (4.10) are:

$$
\left[\begin{array}{ccc}
W^{t} W & a & -I \\
U & 0 & \Lambda \\
a^{t} & 0 & 0
\end{array}\right]\left[\begin{array}{l}
\mathrm{d} \lambda \\
\mathrm{d} \gamma \\
\mathrm{d} \mu
\end{array}\right]=\left[\begin{array}{c}
r_{1} \\
r_{2} \\
r_{3}
\end{array}\right]
$$

where

$$
\begin{gathered}
U=\operatorname{diag}(\mu), \Lambda=\operatorname{diag}(\lambda), \\
r_{1}=\mu-a \gamma-W^{t} W \lambda, \quad r_{2}=-\mu^{t} \lambda, \quad r_{3}=1-a^{t} \lambda .
\end{gathered}
$$

By performing some algebraic manipulations, the directions $\mathrm{d} \mu, \mathrm{d} \lambda$ and $\mathrm{d} \gamma$ are given by:

$$
\begin{aligned}
& \mathrm{d} \mu=\Lambda^{-1} r_{2}-\Lambda^{-1} U \mathrm{~d} \lambda, \\
& \mathrm{d} \lambda=\left(W^{t} W+\Lambda^{-1} U\right)^{-1} r_{4}-\left(W^{t} W+\Lambda^{-1} U\right)^{-1} a \mathrm{~d} \gamma, \\
& a^{t}\left(W^{t} W+\Lambda^{-1} U\right)^{-1} a \mathrm{~d} \gamma=a^{t}\left(W^{t} W+\Lambda^{-1} U\right)^{-1} r_{4}-r_{3},
\end{aligned}
$$

where $r_{4}=r_{1}+\Lambda^{-1} r_{2}$.

Defining $s l 1=\left(W^{t} W+\Lambda^{-1} U\right)^{-1} a$ and $s l 2=\left(W^{t} W+\right.$ $\left.\Lambda^{-1} U\right)^{-1} r_{4}$, to compute the directions the following linear systems must be solved:

$$
\left(W^{t} W+\Lambda^{-1} U\right) s l 1=a,\left(W^{t} W+\Lambda^{-1} U\right) s l 2=r_{4}
$$

Note that $\left(W^{t} W+\Lambda^{-1} U\right)$ is a positive definite matrix of order $p+1$ and both systems can be solved using the same factorization.

\subsection{Theoretical Properties of the Optimal Adjustment Algorithm for $\boldsymbol{p}$ Coordinates}

Theorems 1 and 2, described below and proved in [6], ensure that the optimal adjustment algorithm for $p$ coordinates converge in the worst case with the same convergence rate of von Neumann's algorithm and that, from the theoretical point of view, for larger values of $p$ the algorithm is more robust and will perform better.

Theorem 1 The decrease in $\left\|b^{k}\right\|$ obtained by an iteration of the optimal adjustment algorithm for $p$ coordinates, with $1 \leq p \leq n$, where $n$ is the column number of matrix $P$, in the worst case, is equal to the decrease obtained by an iteration of von Neumann's algorithm.

Theorem 2 The decrease in $\left\|b^{k}\right\|$ obtained by an iteration of the optimal adjustment algorithm for $p_{2}$ coordinates, in the worst case is equal to that obtained by an iteration of the optimal adjustment algorithm for $p_{1}$ coordinates with $p_{1} \leq p_{2} \leq n$, where $n$ is the $P$ column number.

On the other hand, it is not advisable to choose a very large value of $p$ since there is a cost of building and updating matrix $W$ in (4.13). Such a cost is negligible for small values of $p$. However, it becomes noticeable for larger values.

\section{Mehrotra's Heuristic and Starting Point in Interior Point Methods}

It is well known that the starting point can influence the performance of interior point methods. On the other hand, as the optimal adjustment algorithm for $p$ coordinates has a small computational cost per iteration, a natural idea is to use this algorithm to find a good starting point for interior point methods or improve the current one. In this work, few iterations of the optimal adjustment algorithm for $p$ coordinates are performed to improve the starting point introduced by Mehrotra's heuristic [7]. This heuristic consists of the following steps:

1) Use least squares to compute the points:

$$
\tilde{y}=\left(A A^{t}\right)^{-1} A c, \tilde{z}=c-A^{t} \tilde{y}, \tilde{x}=A^{t}\left(A A^{t}\right)^{-1} b
$$

where $x, y$ and $z$ are the primal variables, dual variables and dual slacks respectively.

2) Find values $\delta x$ and $\delta z$ such that $\tilde{x}+\delta_{x}$ and $\tilde{z}+\delta_{z}$ are non-negative:

$\delta_{x}=\max \left(-1.5 \min \left\{\tilde{x}_{i}\right\}, 0\right), \delta_{z}=\max \left(-1.5 \min \left\{\tilde{z}_{i}\right\}, 0\right)$.

3) Determine $\tilde{\delta}_{x}$ and $\tilde{\delta}_{z}$ such that the points $x^{0}$ and 
$z^{0}$ are centralized:

$$
\begin{aligned}
& \tilde{\delta}_{x}=\delta_{x}+\frac{\left(\tilde{x}+\delta_{x} e\right)^{t}\left(\tilde{z}+\delta_{z} e\right)}{2 \sum_{i=1}^{n}\left(\tilde{z}_{i}+\delta_{z}\right)}, \\
& \tilde{\delta}_{z}=\delta_{z}+\frac{\left(\tilde{x}+\delta_{x} e\right)^{t}\left(\tilde{z}+\delta_{z} e\right)}{2 \sum_{i=1}^{n}\left(\tilde{x}_{i}+\delta_{x}\right)} .
\end{aligned}
$$

4) Compute the starting points as follows:

$$
y^{0}=\tilde{y}, z^{0}=\tilde{z}+\tilde{\delta}_{z} e, x^{0}=\tilde{x}+\tilde{\delta}_{x} e .
$$

Remark 5.1 Any linear programming problem can be reduced to problem (1.1). For details of this transformation see [4].

Usually, to improve the performance of interior point methods, modifications are introduced after Step 4 of the algorithm described above, unlike our proposal, which consists of performing a few iterations of the optimal adjustment algorithm for $p$ coordinates before centralizing, i.e., before Step 2. One explanation for this is that if the algorithm is then used after to centralize the points, these are improved. However, the centrality, which is important for the interior point methods, may be lost.

We have adapted the optimal adjustment algorithm for $p$ coordinates in the $\mathrm{PCx}$ code [13] aiming to improve the starting point given by Mehrotra [7].

The starting point for the optimal adjustment algorithm for $p$ coordinates is determined by solving the least squares in Step 1.

\section{Computational Experiments}

In the following experiments, all testing was performed on an Intel Core 2 Duo T7250, 2 GB RAM, 2 GHz and $250 \mathrm{~GB}$ hd and Ubuntu 32-bit operating system. We used 76 test problems to compare the performance of the original PCx and the modified PCx (PCxMod), which uses the optimal adjustment algorithm for $p$ coordinates as a warm-starting approach. Most tested problems have free access such as NETLIB problems, QAPLIB problems and KENNINGTON problems ([14,15]). Other problems, which are not available publicly, were obtained from Gonçalves [4]. The test problems are pre-

\begin{tabular}{|c|c|c|c|c|c|c|c|c|}
\hline Problem & Rows & Columns & Problem & Rows & Columns & Problem & Rows & Columns \\
\hline \multicolumn{3}{|c|}{ NETLIB } & \multicolumn{3}{|c|}{ Miscellaneous } & \multicolumn{3}{|c|}{ Gonçalves } \\
\hline 80bau3b & 2140 & 11066 & nug06 & 372 & 486 & bl & 5729 & 12462 \\
\hline agg2 & 514 & 750 & nug07 & 602 & 931 & bl2 & 5729 & 12462 \\
\hline agg3 & 514 & 750 & nug08 & 912 & 1632 & $\operatorname{co5}$ & 4849 & 10787 \\
\hline czprob & 671 & 2779 & cre-a & 2994 & 6692 & $\operatorname{co9}$ & 9090 & 19997 \\
\hline degen3 & 1503 & 2604 & cre-b & 5336 & 36382 & cq9 & 8004 & 19317 \\
\hline dfl001 & 5984 & 12143 & cre-c & 2375 & 5412 & ex01 & 235 & 1556 \\
\hline etamacro & 334 & 669 & cre-d & 4102 & 28601 & ex02 & 227 & 1548 \\
\hline fffff800 & 322 & 826 & ken-11 & 10085 & 16740 & ex05 & 832 & 7805 \\
\hline fit2d & 25 & 10524 & ken-13 & 22534 & 36561 & ex06 & 825 & 7797 \\
\hline fit2p & 3000 & 13525 & ken-18 & 78862 & 128434 & ex09 & 1821 & 18184 \\
\hline maros-r7 & 2152 & 7440 & osa-07 & 1081 & 25030 & fort45 & 1152 & 1582 \\
\hline modszk1 & 665 & 1599 & osa-14 & 2300 & 54760 & fort 47 & 1152 & 1582 \\
\hline perold & 593 & 1389 & osa-30 & 4313 & 104337 & fort 48 & 1152 & 1582 \\
\hline pilot & 1368 & 4543 & osa-60 & 10243 & 243209 & fort53 & 1156 & 1586 \\
\hline pilotwe & 701 & 2814 & chr25a & 8149 & 15325 & fort58 & 1156 & 1586 \\
\hline scfxm3 & 915 & 1704 & chr22b & 5587 & 10417 & fort59 & 1156 & 1586 \\
\hline seba & 448 & 901 & scr15 & 2234 & 6210 & fort60 & 1156 & 1586 \\
\hline ship081 & 470 & 3121 & scr20 & 5079 & 15980 & fort61 & 1156 & 1586 \\
\hline stocfor3 & 15362 & 22228 & rou20 & 7359 & 37640 & ge & 9150 & 14990 \\
\hline \multirow[t]{9}{*}{ wood1p } & 171 & 1718 & pds-02 & 2609 & 7339 & nl & 6665 & 14680 \\
\hline & & & pds-20 & 32287 & 106080 & $\mathbf{x 1}$ & 1050 & 1480 \\
\hline & & & pds-30 & 47968 & 156042 & $\mathrm{x} 2$ & 1050 & 1480 \\
\hline & & & pds-40 & 64276 & 214385 & & & \\
\hline & & & pds-50 & 80339 & 272513 & & & \\
\hline & & & pds-60 & 96514 & 332862 & & & \\
\hline & & & pds-70 & 111896 & 386238 & & & \\
\hline & & & pds-80 & 126120 & 430800 & & & \\
\hline & & & pds-90 & 139752 & 471538 & & & \\
\hline
\end{tabular}
sented in Table 1.

Table 1. Test problems. 


\subsection{Choice of $p$}

For the optimal adjustment algorithm for $p$ coordinates to work properly an appropriate choice of parameter $p$ is essential. Thus, several computational experiments were done to determine a heuristic that works well in any linear programming problem. With results obtained in the tests, it became clear that the value of $p$ must be chosen depending on the size of the problem. Recalling that $m$ is the number of rows and $n$ is the number of columns of the linear problem constraint matrix, the heuristic that showed better results is as follows:

\begin{tabular}{c}
\hline $0<(m+n) \leq 10,000 \rightarrow 0$ - $p=4$ \\
$10,000<(m+n) \leq 20,000 \rightarrow p=8$ \\
$20,000<(m+n) \leq 400,000 \rightarrow p=20$ \\
$400,000<(m+n) \leq 600,000 \rightarrow p=40$ \\
$600,000<(m+n)$ \\
\hline
\end{tabular}

\subsection{Stopping Criterion}

The number of iterations of the optimal adjustment algorithm for $p$ coordinates to be performed is an important parameter to be determined, since it directly influences the performance of the PCx. This algorithm achieves better results when the solution is determined with good accuracy. However, in some cases, the number of iterations needed for convergence of the algorithm can be very large, making it impractical to use. In these cases, a maximum number of iterations should be adopted. Therefore, the stopping criterion for the optimal adjustment algorithm for $p$ coordinates used is the following: maximum number of iterations (100) or the relative error of the residual norm smaller than a given tolerance $\left(10^{-4}\right)$ (the one that occurs first).

\subsection{Results}

The performance of the PCx and PCxMod was compared with respect to the total number of iterations and the run time. The results are presented in Table 2.

The total number of interior point iterations (Column Iterations) and the total running time in seconds (Column Time) of two versions of the PCx are showed in Table 2. The PCxMod is the version that uses the optimal adjustment algorithm for $p$ coordinates in Mehrotra's heuristic and $\mathrm{PCx}$ is the one that does not adopt it. Column $p$ shows the value of $p$ used by the optimal adjustment algorithm. Column ItAux gives the number of iterations performed by the optimal adjustment algorithm for $p$ coordinates.
According to the results, the PCxMod takes less time to obtain the optimal solution in $55.3 \%$ of the tested problems and the PCx in $34.2 \%$ of the problems. Moreover, the total number of iterations was lower in $40.8 \%$ of the problems for the PCxMod and $1.3 \%$ (only one problem) for the PCx. In this case, the run time of the PCxMod was lower because a better starting point was used.

In almost all problems with larger running time, the new approach performed better than the traditional one. This reveals a welcome feature of the proposed approach, since those are the most important problems to be solved.

Although the total number of iterations did not decrease in some problems, a better starting point was determined as the optimal adjustment algorithm for $p$ coordinates was used after Step 1 of the Mehrotra's heuristic and, consequently, the running time was reduced.

An interesting result is the fact that two problems (co9 and nug08) were solved only by PCxMod.

In another experiment, the same set of test problems were solved using only the PCxMod. First, $p=2$ (2-coord) was considered, which represents the optimal pair adjustment algorithm and after the value of $p$ according to the stopping criterion previously described was determined. The results obtained are shown in Table 3.

The results showed that the PCxMod takes less iterations with values of $p$ greater than 2 in $40.8 \%$ of the tests problems. For $p=2$ this number is approximately 5.3\%. The total running time was reduced by about $60.5 \%$ of the problems for $p$-coordinates and only $22.4 \%$ were solved in a lower time as $p=2$ was considered.

Again the problem co9 obtained status optimal only for $p=8$.

These tests confirmed that the performance of the algorithm is improved by increasing the value of $p$. That happens because the residual $b^{k}$ has a greater reduction from one iteration to another and also because the given point by the algorithm as a solution is different for each value of $p$. The obtained points for $p>2$ achieved better performance in most cases.

It should be mentioned that the required total time to obtain a solution for the optimal adjustment algorithm for $p$ coordinates is not significant in relation to the resolution total time of the problems. This time is almost null in many of the tested problems. Considering the pds- 80 problem, the time spent by the optimal adjustment algorithm was 24 seconds, which represents approximately $0.07 \%$ of the total running time.

\section{Conclusions and Future Work}

In this work, the optimal adjustment algorithm for $p$ co- 
Table 2. PCx $\times$ PCxMod.

\begin{tabular}{|c|c|c|c|c|c|c|c|c|}
\hline \multicolumn{5}{|c|}{ Dimension } & \multicolumn{2}{|c|}{ Iterations } & \multicolumn{2}{|c|}{ Time } \\
\hline Problem & Rows & Columns & $\mathbf{p}$ & itAux & PCx & PCxMod & PCx & PCxMod \\
\hline 80bau3b & 2140 & 11066 & 8 & 2 & 36 & 36 & 0.4 & 0.44 \\
\hline agg2 & 514 & 750 & 4 & 2 & 21 & 21 & 0.1 & 0.07 \\
\hline agg3 & 514 & 750 & 4 & 2 & 19 & 19 & 0.12 & 0.05 \\
\hline czprob & 671 & 2779 & 4 & 2 & 26 & 25 & 0.05 & 0.05 \\
\hline degen 3 & 1503 & 2604 & 4 & 10 & 15 & 15 & 0.77 & 0.8 \\
\hline dfl001 & 5984 & 12143 & 8 & 2 & 45 & 41 & 77.27 & 69.27 \\
\hline etamacro & 334 & 669 & 4 & 10 & 26 & 25 & 0.04 & 0.04 \\
\hline fffff800 & 322 & 826 & 4 & 2 & 29 & 28 & 0.07 & 0.07 \\
\hline fit2d & 25 & 10524 & 4 & 2 & 22 & 22 & 0.52 & 0.55 \\
\hline fit2p & 3000 & 13525 & 8 & 2 & 20 & 20 & 0.3 & 0.36 \\
\hline maros-r7 & 2152 & 7440 & 8 & 2 & 16 & 16 & 1.94 & 1.94 \\
\hline modszk1 & 665 & 1599 & 4 & 4 & 20 & 20 & 0.04 & 0.04 \\
\hline perold & 593 & 1389 & 4 & 2 & 32 & 32 & 0.15 & 0.12 \\
\hline pilot & 1368 & 4543 & 4 & 2 & 34 & 30 & 2.06 & 1.93 \\
\hline pilot87 & 1971 & 6373 & 4 & 2 & 25 & 25 & 5.19 & 5.15 \\
\hline pilotwe & 701 & 2814 & 4 & 6 & 45 & 44 & 0.2 & 0.16 \\
\hline scfxm3 & 915 & 1704 & 4 & 2 & 19 & 19 & 0.08 & 0.06 \\
\hline seba & 448 & 901 & 4 & 2 & 13 & 13 & 0.25 & 0.24 \\
\hline ship08I & 470 & 3121 & 4 & 2 & 14 & 14 & 0.06 & 0.04 \\
\hline stocfor3 & 15362 & 22228 & 10 & 2 & 30 & 30 & 1.16 & 1.2 \\
\hline wood1p & 171 & 1718 & 4 & 10 & 22 & 22 & 0.26 & 0.3 \\
\hline cre-a & 2994 & 6692 & 8 & 2 & 23 & 23 & 0.25 & 0.23 \\
\hline cre-b & 5336 & 36382 & 8 & 2 & 35 & 35 & 3.21 & 3.34 \\
\hline cre-c & 2375 & 5412 & 8 & 2 & 25 & 25 & 0.19 & 0.21 \\
\hline cre-d & 4102 & 28601 & 8 & 2 & 35 & 35 & 2.73 & 2.79 \\
\hline ken-11 & 10085 & 16740 & 8 & 2 & 20 & 20 & 0.62 & 0.72 \\
\hline ken-13 & 22534 & 36561 & 10 & 2 & 23 & 23 & 1.89 & 2.24 \\
\hline ken-18 & 78862 & 128434 & 20 & 2 & 26 & 26 & 15.96 & 18.14 \\
\hline osa-07 & 1081 & 25030 & 4 & 2 & 22 & 22 & 0.56 & 0.6 \\
\hline osa-14 & 2300 & 54760 & 8 & 2 & 25 & 25 & 1.65 & 1.74 \\
\hline osa-30 & 4313 & 104337 & 8 & 4 & 24 & 21 & 3.86 & 4.8 \\
\hline osa-60 & 10243 & 243209 & 8 & 5 & 31 & 24 & 15.38 & 17.06 \\
\hline bl & 5729 & 12462 & 8 & 2 & 32 & 32 & 1.48 & 1.54 \\
\hline bl2 & 5729 & 12462 & 8 & 7 & 37 & 35 & 1.69 & 1.71 \\
\hline $\operatorname{co5}$ & 4849 & 10787 & 8 & 2 & 47 & 47 & 1.72 & 1.72 \\
\hline co9 & 9090 & 19997 & 8 & 20 & $*$ & 46 & $*$ & 6.14 \\
\hline cq9 & 8004 & 19317 & 8 & 2 & 46 & 44 & 4.67 & 4.59 \\
\hline ex01 & 235 & 1556 & 4 & 2 & 25 & 25 & 0.12 & 0.1 \\
\hline ex02 & 227 & 1548 & 4 & 3 & 31 & 30 & 0.11 & 0.11 \\
\hline ex05 & 832 & 7805 & 4 & 4 & 32 & 31 & 0.68 & 0.68 \\
\hline ex06 & 825 & 7797 & 4 & 9 & 63 & 58 & 1.17 & 1.16 \\
\hline ex09 & 1821 & 18184 & 4 & 2 & 37 & 36 & 1.61 & 1.63 \\
\hline fort45 & 1152 & 1582 & 4 & 12 & 17 & 15 & 0.09 & 0.08 \\
\hline fort 47 & 1152 & 1582 & 4 & 2 & 16 & 16 & 0.11 & $\begin{array}{l}0.07 \\
0.07\end{array}$ \\
\hline fort48 & 1152 & 1582 & 4 & 10 & 15 & 12 & 0.1 & 0.07 \\
\hline fort53 & 1156 & 1586 & 4 & 3 & 17 & 16 & 0.11 & 0.08 \\
\hline fort56 & 1156 & 1586 & 4 & 3 & 17 & 17 & 0.11 & 0.09 \\
\hline fort58 & 1156 & 1586 & 4 & 3 & 17 & 17 & 0.13 & 0.09 \\
\hline fort59 & 1156 & 1586 & 4 & 3 & 16 & 16 & 0.12 & 0.08 \\
\hline fort60 & 1156 & 1586 & 4 & 19 & 17 & 16 & 0.13 & 0.11 \\
\hline fort61 & 1156 & 1586 & 4 & 19 & 18 & 16 & 0.12 & 0.11 \\
\hline ge & 9150 & 14990 & 8 & 4 & 43 & 37 & 2.17 & 2.1 \\
\hline nl & 6665 & 14680 & 8 & 2 & 33 & 33 & 2.62 & 2.61 \\
\hline $\mathbf{x 1}$ & 1050 & 1480 & 4 & 2 & 10 & 10 & 0.08 & 0.04 \\
\hline $\mathrm{x} 2$ & 1050 & 1480 & 4 & 10 & 20 & 16 & 0.07 & 0.08 \\
\hline els19 & 4350 & 13186 & 8 & 10 & 20 & 20 & 145.35 & 144.32 \\
\hline chr25a & 8149 & 15325 & 8 & 10 & 23 & 21 & 40.65 & 37.08 \\
\hline chr22b & 5587 & 10417 & 8 & 10 & 21 & 21 & 15.58 & 15.79 \\
\hline scr15 & 2234 & 6210 & 8 & 10 & 16 & 16 & 22.48 & 22.69 \\
\hline scr20 & 5079 & 15980 & 8 & 2 & 14 & 15 & 281.85 & 266.86 \\
\hline
\end{tabular}




\begin{tabular}{|c|c|c|c|c|c|c|c|c|}
\hline rou20 & 7359 & 37640 & 8 & 2 & 13 & 13 & 1524.12 & 1459.71 \\
\hline nug06 & 372 & 486 & 4 & 4 & 21 & 10 & 0.19 & 0.08 \\
\hline nug07 & 602 & 931 & 4 & 2 & 9 & 9 & 0.26 & 0.23 \\
\hline nug08 & 912 & 1632 & 4 & 3 & * & 7 & * & 0.76 \\
\hline pds-02 & 2609 & 7339 & 8 & 2 & 24 & 24 & 0.25 & 0.28 \\
\hline pds-06 & 9156 & 28472 & 8 & 10 & 29 & 29 & 8.18 & 8.49 \\
\hline pds-10 & 15648 & 48780 & 10 & 2 & 33 & 33 & 42.51 & 39.14 \\
\hline pds-20 & 32287 & 106080 & 20 & 2 & 43 & 39 & 407.83 & 392.01 \\
\hline pds-30 & 47968 & 156042 & 20 & 9 & 43 & 42 & 1369.47 & 1318.48 \\
\hline pds-40 & 64276 & 214385 & 20 & 2 & 50 & 50 & 4506.78 & 4644.72 \\
\hline pds-50 & 80339 & 272513 & 20 & 2 & 52 & 51 & 8406.11 & 8301.49 \\
\hline pds-60 & 96514 & 332862 & 20 & 10 & 52 & 50 & 14293.89 & 13687.98 \\
\hline pds-70 & 111896 & 386238 & 20 & 2 & 55 & 55 & 24542.93 & 23943.35 \\
\hline pds-80 & 126120 & 430800 & 20 & 10 & 54 & 51 & 33795.18 & 32035.45 \\
\hline pds-90 & 139752 & 471538 & 20 & 2 & 52 & 51 & 37007.94 & 36638.86 \\
\hline pds-100 & 152300 & 498530 & 40 & 2 & 57 & 57 & 47793.96 & 48147.86 \\
\hline
\end{tabular}

*: means that the method failed.

Table 3. 2-coordinates $\times p$-coordinates.

\begin{tabular}{|c|c|c|c|c|c|c|c|c|c|}
\hline \multirow[b]{2}{*}{ Problem } & \multicolumn{2}{|c|}{ Dimension } & \multirow[b]{2}{*}{$\mathbf{p}$} & \multicolumn{2}{|c|}{ ItAux } & \multicolumn{2}{|c|}{ Iterations } & \multicolumn{2}{|c|}{ Time } \\
\hline & Rows & Columns & & 2 -coord & p-coord & 2 -coord & p-coord & 2 -coord & p-coord \\
\hline 80bau3b & 2140 & 11066 & 8 & 2 & 2 & 36 & 36 & 0.43 & 0.44 \\
\hline agg2 & 514 & 750 & 4 & 2 & 2 & 21 & 21 & 0.07 & 0.07 \\
\hline agg3 & 514 & 750 & 4 & 2 & 2 & 19 & 19 & 0.06 & 0.05 \\
\hline czprob & 671 & 2779 & 4 & 2 & 2 & 25 & 25 & 0.06 & 0.05 \\
\hline degen3 & 1503 & 2604 & 4 & 2 & 10 & 16 & 15 & 0.82 & 0.8 \\
\hline dfl001 & 5984 & 12143 & 8 & 2 & 2 & 42 & 41 & 72.53 & 69.27 \\
\hline etamacro & 334 & 669 & 4 & 2 & 10 & 26 & 25 & 0.04 & 0.04 \\
\hline fffff800 & 322 & 826 & 4 & 6 & 2 & 28 & 28 & 0.08 & 0.07 \\
\hline fit2d & 25 & 10524 & 4 & 2 & 4 & 22 & 22 & 0.55 & 0.55 \\
\hline fit2p & 3000 & 13525 & 8 & 2 & 2 & 20 & 20 & 0.33 & 0.36 \\
\hline maros-r7 & 2152 & 7440 & 8 & 2 & 2 & 12 & 16 & 2.31 & 1.94 \\
\hline modszk1 & 665 & 1599 & 4 & 4 & 4 & 20 & 20 & 0.05 & 0.04 \\
\hline perold & 593 & 1389 & 4 & 2 & 2 & 32 & 32 & 0.12 & 0.12 \\
\hline pilot & 1368 & 4543 & 4 & 2 & 2 & 34 & 30 & 2.09 & 1.93 \\
\hline pilot87 & 1971 & 6373 & 4 & 2 & 2 & 25 & 25 & 5.23 & 5.15 \\
\hline pilotwe & 701 & 2814 & 4 & 2 & 6 & 45 & 44 & 0.18 & 0.16 \\
\hline scfxm3 & 915 & 1704 & 4 & 2 & 2 & 19 & 19 & 0.06 & 0.06 \\
\hline seba & 448 & 901 & 4 & 2 & 2 & 13 & 13 & 0.28 & 0.24 \\
\hline ship08I & 470 & 3121 & 4 & 2 & 2 & 14 & 14 & 0.03 & 0.04 \\
\hline stocfor3 & 15362 & 22228 & 10 & 2 & 2 & 30 & 30 & 1.2 & 1.2 \\
\hline wood1p & 171 & 1718 & 4 & 2 & 10 & 22 & 22 & 0.27 & 0.3 \\
\hline cre-a & 2994 & 6692 & 8 & 2 & 2 & 23 & 23 & 0.22 & 0.23 \\
\hline cre-b & 5336 & 36382 & 8 & 2 & 2 & 35 & 35 & 3.36 & 3.34 \\
\hline cre-c & 2375 & 5412 & 8 & 2 & 2 & 25 & 25 & 0.22 & 0.21 \\
\hline cre-d & 4102 & 28601 & 8 & 2 & 2 & 35 & 35 & 2.81 & 2.79 \\
\hline ken-11 & 10085 & 16740 & 8 & 6 & 2 & 20 & 20 & 0.73 & 0.72 \\
\hline ken-13 & 22534 & 36561 & 10 & 2 & 2 & 23 & 23 & 2.03 & 2.24 \\
\hline ken-18 & 78862 & 128434 & 20 & 2 & 2 & 29 & 26 & 15.63 & 18.14 \\
\hline osa-07 & 1081 & 25030 & 4 & 2 & 2 & 22 & 22 & 0.6 & 0.6 \\
\hline osa-14 & 2300 & 54760 & 8 & 2 & 2 & 25 & 25 & 1.74 & 1.74 \\
\hline osa-30 & 4313 & 104337 & 8 & 2 & 4 & 21 & 21 & 4.32 & 4.8 \\
\hline osa-60 & 10243 & 243209 & 8 & 2 & 5 & 31 & 24 & 15.87 & 17.06 \\
\hline bl & 5729 & 12462 & 8 & 2 & 2 & 32 & 32 & 1.5 & 1.54 \\
\hline bl2 & 5729 & 12462 & 8 & 2 & 7 & 37 & 35 & 1.69 & 1.71 \\
\hline $\operatorname{co5}$ & 4849 & 10787 & 8 & 2 & 2 & 47 & 47 & 1.72 & 1.72 \\
\hline $\operatorname{co9}$ & 9090 & 19997 & 8 & & 20 & & 46 & & 6.14 \\
\hline cq9 & 8004 & 19317 & 8 & 2 & 2 & 45 & 44 & 4.62 & 4.59 \\
\hline ex01 & 235 & 1556 & 4 & 2 & 2 & 25 & 25 & 0.11 & 0.1 \\
\hline ex02 & 227 & 1548 & 4 & 2 & 3 & 31 & 30 & 0.16 & 0.11 \\
\hline ex05 & 832 & 7805 & 4 & 2 & 4 & 32 & 31 & 0.68 & 0.68 \\
\hline ex06 & 825 & 7797 & 4 & 2 & 9 & 64 & 58 & 1.22 & 1.16 \\
\hline
\end{tabular}




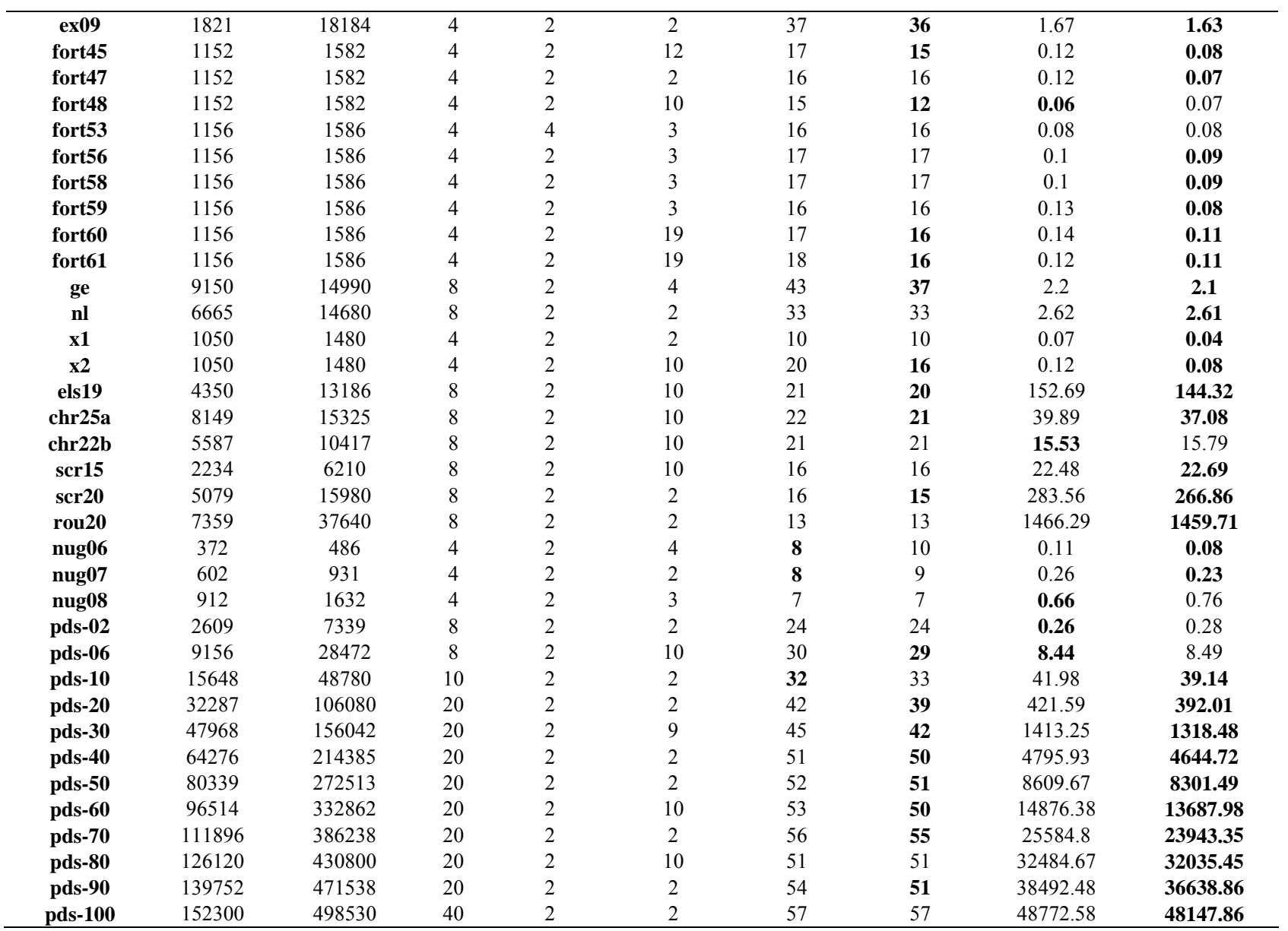

*: means that the method failed.

ordinates was used in conjunction with the interior point method to determine good starting points, enabling the method to perform better and consequently converge faster. The main advantages of this algorithm are simplicity and quick initial progress.

Numerical experiments on a set of problems showed that by using this approach it is possible to reduce the total number of iterations and the running time for many problems, mainly large-scale ones. In addition, two problems were solved to optimality only by this approach. That is, using the algorithm for $p$ coordinates to improve the starting point computed by PCx leads to a more robust implementation.

By incorporating the optimal adjustment algorithm for $p$ coordinates on the code PCx, more specifically Mehrotra's heuristic, and performing a few iterations, a more robust implementation was obtained.

The computational experiments on a set of linear programming problems showed that this approach can reduce the total number of iterations and total run time for several problems, especially the larger problems and those with higher running time. Moreover, the optimal solution of some unsolved problems was found.

As future work, sophisticated heuristics for the choice of the number of coordinates $p$ will be developed as after several experiments, values for $p$ that reduce the number of iterations by about $90 \%$ of the test problems were found. Also other stopping criterion for the optimal adjustment algorithm for $p$ coordinates will be investigated, since the number of iterations performed by such an algorithm directly influences the quality of the starting point determined. New forms of choosing the $p$ columns will be developed.

\section{Acknowledgements}

This research was sponsored by the Brazilian Agencies CAPES and CNPq.

\section{References}

[1] G. B. Dantzig, "Converting A Converging Algorithm into a Polynomially Bounded Algorithm," Technical Report, Stanford University, SOL 91-5, 1991. 
[2] G. B. Dantzig, "An $\in$-Precise Feasible Solution to a Linear Program with a Convexity Constraint in $1 / \epsilon^{2}$ Iterations Independent of Problem Size," Technical Report, Stanford University, SOL 92-5, 1992.

[3] M. Epelman and R. M. Freund, "Condition Number Complexity of an Elementary Algorithm for Computing a Reliable Solution of a Conic Linear System," Mathematical Programming, Vol. 88, No. 3, 2000, pp. 451-485.

[4] J. P. M. Gonçalves, "A Family of Linear Programming Algorithms Based on the Von Neumann Algorithm," Ph.D. Thesis, Lehigh University, Bethlehem, 2004.

[5] J. P. M. Gonçalves, R. H. Storer and J. Gondzio, "A Family of Linear Programming Algorithms Based on an Algorithm by Von Neumann," Optimization Methods and Software, Vol. 24, No. 3, 2009, pp. 461-478. doi:10.1080/10556780902797236

[6] J. Silva, "Uma Família de Algoritmos para Programação Linear Baseada no Algoritmo de Von Neumann," Ph.D. Thesis, IMECC-UNICAMP, Campinas, 2009 (in Portuguese).

[7] S. Mehrotra, "On the Implementation of a Primal-Dual Interior Point Method," SIAM Journal on Optimization, Vol. 2, No. 4, 1992, pp. 575-601.

[8] H. Y. Benson and D. F. Shanno, "An Exact Primal Dual Penalty Method Approach to Warm Starting InteriorPoint Methods for Linear Programming," Computational
Optimization and Applications, Vol. 38, No. 3, 2007, pp. 371-399. doi:10.1007/s10589-007-9048-6

[9] E. John and E. A. Yildirim, "Implementation of WarmStart Strategies in Interior-Point Methods for Linear Programming in Fixed Dimension," Computational Optimization and Applications, Vol. 41, No. 2, 2008, pp. 151183. doi:10.1007/s10589-007-9096-y

[10] A. Engau, M. F. Anjos and A. Vannelli, "A Primal-Dual Slack Approach to Warm Starting Interior-Point Methods for Linear Programming," Operations Research and Cyber-Infrastructure, Vol. 47, 2009, pp. 195-217. doi:10.1007/978-0-387-88843-9 10

[11] G. B. Dantzig and M. N. Thapa, "Linear Programming 2: Theory and Extensions," Springer-Velag, New York, 1997.

[12] D. Bertsimas and J. N. Tsitsiklis, "Introduction to Linear Optimization," Athena Scientific, Belmont, 1997.

[13] J. Czyzyk, S. Mehrotra, M. Wagner and S. J. Wright, "PCx an Interior Point Code for Linear Programming," Optimization Methods \& Software, Vol. 11, No.1-4, 1999, pp. 397-430. doi:10.1080/10556789908805757

[14] NETLIB Collection LP Test Sets, "NETLIB LP Repository". http://www.netlib.org/lp/data

[15] M. L. Models, "Hungarian Academy of Sciences OR Lab". http://www.sztaki.hu/meszaros/puplic-ftp/lptestset/mist 